BOOK REVIEW

\section{Angels of Death: Exploring the Euthanasia Underground}

R S Magnusson, Melbourne University Press, 2002. ISBN 0-522-84970-9

Angels of Death, which reports on Magnusson's study of the euthanasia underground within the HIV/AIDS communities principally in Sydney, Melbourne, and San Francisco, is, in many respects, a unique work. It is written by a legal scholar but is quite deliberately non-legalistic; indeed, Magnusson makes clear his intention is not to create another manifesto but to inject new perspectives into the euthanasia debate. The book's underlying methodology also sets it apart. It is based on the author's own extensive empirical research, drawing on data gathered over three years in the course of indepth, face to face interviews with healthcare workers in Australia and the USA who have been involved in caring for people with AIDS. This kind of empirical research is not often undertaken by lawyers, and while a number of studies into attitudes and practices of healthcare professionals in relation to euthanasia have been carried out by researchers from other disciplines, very few have involved interview based research, relying instead predominantly on surveys. ${ }^{1}$ By any measure, Magnusson's research has been undertaken with meticulous care. He is able to balance his gratitude and respect for his interviewees with the need to provide a dispassionate and evenhanded commentary on their accounts.

The aim of Magnusson's study is to investigate the attitudes and practices of Australian and American healthcare workers towards assisted suicide and euthanasia especially within HIV/AIDS healthcare contexts. While this presents some atypical features from other healthcare contexts where assisted death is an issue (which may, in turn, have implications for the extent to which we can extrapolate from the book's findings in relation to the "euthanasia underground"), it serves as a useful focal point for this study. The book presents and analyses first person accounts of involvement in assisted suicide and euthanasia and contextualises interviewees' involvement in assisted death within the broader context of palliative care. The author's intention in telling these stories of the "angels of death" "is to explore that secret part of the medical and nursing professions that few will ever talk about, and about which the public knows very little". ${ }^{\prime 2}$ In turn, the book's purpose is stated to be "to expose the social practices, relationships, and networks that constitute 'underground' euthanasia" with a view to demonstrating the complexity of the issue and capturing some of the "passion, pathos and bittersweetness of those bedside moments". ${ }^{3}$ This is an ambitious objective but one which is richly fulfilled.

It is crucial that the development of law and policy on the important issue of euthanasia is based on an informed understanding of actual practice. This empirical work, through the collation and evaluation of indepth interviews, makes an important contribution to the euthanasia debate. This is particularly the case in the light of the limitations of earlier empirical work performed in Australia. The most substantia study to be carried out to date is that by Kuhse et al amongst Australian medical practitioners to assess their attitudes to and practices in relation to euthanasia, ${ }^{4}$ modelled on a major Dutch study. ${ }^{5}$ Significantly however, the researchers had not been able to undertake the interview component of the research. This was reported to be due to concerns about the potential legal liability of respondents and the need to maintain anonymity. ${ }^{6}$ (In the corresponding Dutch study, participating doctors had been granted immunity from liability.) Therefore, the study by Kuhse et al had relied exclusively on anonymous surveys. As Magnusson points out, "while survey-based studies can monitor trends in attitudes and actions, they do not facilitate a detailed exploration of the processes that operate at the bedside, and the social and emotional milieu within which assisted suicide and euthanasia are practised". ${ }^{7}$ This limitation was overcome in Magnusson's work where interviews were conducted on a pseudonymous basis in accordance with a written protocol which had been approved by the Human Research Ethics Committee of the University of Melbourne. No master list of interviewees was retained, thereby preserving the anonymity of respondents. Thus, although all accounts were self-incriminating and could potentially lead to serious criminal liability, the study was designed in a way to ensure that there can be no identification of individual respondents. Moreover, as Magnusson notes, this assurance of anonymity has facilitated unprecedented access to the world of illicit euthanasia.

As one would expect, much of the book is devoted to presenting and exploring the accounts of the interviewees in this study. Chapter 1 entitled "Who would do such a thing?" examines who performs illegal euthanasia. It profiles five "euthanisers", presenting some of the anecdotes, personalities, and personal philosophies that emerged from the interviews. It is short but confronting, highlighting the diversity of the individuals who take this path and their approach to euthanasia. The next few chapters seek to put these accounts into some context, examining the social and legal dimensions of the debate about assisted death. Chapter 2 surveys "doctors who kill", setting out some of the reported accounts of doctor participation in euthanasia in Australia, the UK, and the USA. Chapter 3 focuses on the most prominent of the "voices" and issues in the euthanasia debate and looks also at the impact of AIDS on euthanasia advocacy. Chapter 4 explores some of the factors behind the demise of the "sanctity of life" idea and then reviews, under the heading "Experimenting with death", some of the legislative models that have been implemented, including the now defunct Northern Territory Rights of the Terminally Ill Act 1995 , Oregon's Death with Dignity Act of 1994 which has legalised physician assisted suicide, and recent legislative developments in the Netherlands pursuant to which the longstanding defence of necessity for doctors who perform euthanasia in accordance with due care requirements has now been given an explicit statutory basis. ${ }^{8}$

The book then returns to the interviewees and the "euthanasia underground" Chapter 5 investigates the meaning of "suicide talk" and highlights the great variety of factors that motivate requests for assistance. This is then followed in Chapter 6 (which also contains contributions from a collaborator, P H Ballis) with an exploration of the options that health carers have in responding to these requests. Chapter 7 consolidates the data from the study and presents some of the major themes and findings. It also acknowledges the limitations of the interview methodology employed, constrained as it is by what interviewees are willing to divulge. Moreover, Magnusson expressly recognises that methodology can be an easy target for studies which present "uncomfortable findings" and indeed, flags as one of a number of possible responses to the euthanasia underground to "attack (or ignore) the messenger". 9 In an effort to anticipate and silence possible critics, the following chapter, "Doing fieldwork in the euthanasia underground", (also with contributions from $\mathrm{P} \mathrm{H}$ Ballis) seeks to make the interview event as transparent as possible, reflecting on what interviewees said about what they did, how they said it, and what they achieved by saying it. In so doing, the authors seek to illustrate and defend what they believe remains a successful model for conducting fieldwork into concealed and underground phenomena. Chapter 9, "The underground community", presents fascinating insights into the network constituted by the informal chain of associations between health carers who approve of, facilitate, and participate in assisted death. This is followed by two related chapters which seek to present a number of "disturbing issues" arising from the study, centring on the lack of medical professionalism. ${ }^{10}$ These are arguably the most important chapters in the whole book. In Chapter 12, "De-mystifying euthanasia practice", the author draws on some of the themes of previous chapters to interrogate some "takenfor granted assumptions and misconceptions about euthanasia practice" ${ }^{\prime 1}$ " He is no doubt correct in suggesting that ultimately, a demystified perspective on euthanasia is one which acknowledges the complex, multifaceted, and highly personalised context within which end of life decisions are made. The book concludes with a chapter canvassing possible policy responses to the euthanasia underground. Magnusson's preferred approach, albeit one which he puts forward somewhat guardedly, is to legalise, and attempt to regulate, the practice of euthanasia. He is quite candid, however, in stating that his initial confidence about the correctness of this position has, after continuing reflection, been tempered with concern about "the potential, barely tangible risks of legalisation ...".

What does this book tell us? It is hardly a surprise that a euthanasia underground exists-we already knew this much from earlier survey based empirical data. These data had identified a practice of active voluntary euthanasia at a rate comparable with that in the Netherlands (where there has for many years been legal acceptance of the practice if performed under certain conditions), and an alarming rate of active termination of life without an explicit request-almost three times the rate in the Netherlands. ${ }^{12}$ What this book achieves is to give insight into the circumstances in which euthanasia and assisted suicide are performed, drawing on the real life experiences and perspectives of the respondents. It maps the highs and lows of these experiences, faithfully recounting the stories of the participants. What the book also does is to highlight how much we don't know about 
these practices-although the focus is usually on doctors, and to a lesser extent nurses, a whole range of healthcare professionals and others appear to be involved. In this study, the 49 respondents, all of whom recounted personal experience with performing euthanasia and/or assisted suicide, comprised 19 doctors (including psychiatrists), 17 nurses, 7 therapists (psychologists/counsellors), 5 community workers and a funeral director, the latter having reported that the informal network had been dubbed "Angels of Death".) The book also draws attention to the fragmentation of opinion within the health professions on the issue of assisted death.

We need to take some important lessons from the book's spotlight on these disturbing issues. Whilst Magnusson suggests that the findings of the book provide "ammunition" for both sides of the euthanasia debate, they seems to stack up very much against the present policy of prohibition. In any event, strictly speaking, the study findings provide no evidence in relation to a policy of regulation as this has not (with the exception of a short period in the Northern Territory which was not the subject of this study) been attempted in Australia. Moreover, although euthanasia opponents are quick to rely on the slippery slope mantra, as Magnusson acknowledges, the risk of a slippery slope remains to be demonstrated. ${ }^{13}$

When one surveys the "disturbing issues" that emerge from his research, it is the absence of professionalism which, above all, characterises the euthanasia underground. There are numerous aspects to this, including the lack of specialised training and widespread ignorance about what is needed to achieve a gentle death, with frequent "botched" attempts; the lack of accountability or norms or principles guiding involvement - with evidence of coercion upon the patient and/or the healthcare worker, evidence of rash or hasty involvement by doctors with little or no knowledge of the patient's circumstances, and an all pervasive culture of deception. Particularly troubling is the evidence of non-consensual euthanasia. While this is not really unexpected (confirming the findings of earlier studies such as that carried out by Kuhse et $\mathrm{al}^{14}$ ), the detailed accounts of individual experiences provide a chilling insight into what happens when health carers take it upon themselves to make these decisions. What is notable, are the differences between the results of Magnusson's study and that of Kuhse et al: Magnusson states (at p 232) that voluntary euthanasia was the dominant form of euthanasia practised by interviewees, whereas the Kuhse et al study had found that the incidence in Australia of killing by doctors without request was significantly higher than for active voluntary euthanasia (3.5\% of all deaths compared with $1.8 \%$ for euthanasia). A number of possible explanations may be advanced for this discrepancy all of which are linked with the special characteristics of the subgroup that Magnusson has focused his study on: the candidates are typically relatively young, articulate, and well connected. This brings us back to the point made earlier that the atypical features of the HIV/AIDS healthcare context possibly limits the extent to which we can extrapolate from the book's findings to the wider euthanasia context. Nevertheless, the HIV/AIDS context is clearly indicative of what occurs and thereby serves as a useful window into the hidden practice of euthanasia in Australia.

In a society which values autonomy, it is this evidence of non-consensual euthanasia which, above all, demands reassessment of the prevailing policy of prohibition. As Magnusson concludes, prohibition has been ineffective to inhibit the practice of euthanasia or to adequately protect vulnerable patients. If we are to adopt Magnusson's starting premise of a "harm minimisation approach to euthanasia policy", ${ }^{15}$ one must factor into the equation evidence from other studies which suggests that there is a greater risk of patients' lives being taken without their explicit consent in countries such as Australia (where euthanasia has been prohibited) than in the Netherlands where euthanasia has been legal, at least in a de facto sense, for many years. ${ }^{16}$ This would appear to provide quite compelling grounds to intervene and regulate for the protection of patients. As Magnusson recognises, the choice we face is not a choice between having no euthanasia and making euthanasia legal: rather it is a choice between driving it underground (with all the related concerns about lack of transparency and medical professionalism), and making it visible. The importance of also protecting the autonomy and voluntariness of healthcare professionals should not be overlooked.

Justice Kirby, in his erudite foreword to Angels of Death, describes it as a "troubling book with an upsetting title about a disturbing topic". It is unquestionably a profound and thought provoking work which makes an invaluable contribution to the euthanasia debate. Indeed, as Justice Kirby suggests, after this book, the debate will never be the same. Measured against the author's own stated aims, the book succeeds in injecting new perspectives into that debate and exposing the complexity of the practices, relationships, and networks that constitute the "euthanasia underground".

Angels of Death is an exceptionally readable work and would be engaging for anyone with an interest in the issue of euthanasia. Magnusson's book helps to instil a high level of reality into the euthanasia controversy with its rich accounts of the experiences of those at the "coal face": and while the message that the book delivers is not always a palatable one, indeed, at times perturbing, it is impossible to ignore. Angels of Death is an important and powerful work which deservedly has received international interest. It is hoped that appropriate notice is taken of its message in Australia to force reassessment of current law and policy on this difficult and challenging issue.

M Otlowski

\section{References}

1 For example, Kuhse H, Singer P. Doctors practices and attitudes regarding voluntary euthanasia. Med J Aust 1988:148:623-7. Baume P, O'Malley E. Euthanasia: attitudes and practices of medical practitioners. Med I Aust 1994; 161: 137, 140, 142-4; Kuhse H, Singer P, Baume $\mathrm{P}$, et al. End of life decisions in Australian medical practice. Med J Aust, 1997:166:191-6.

2 Magnusson RS. Angels of Death: Exploring the Euthanasia Underground. Melbourne University Press, 2002:2

3 See reference 2: 5 .

4 Kuhse $\mathrm{H}$, Singer $\mathrm{P}$, Baume $\mathrm{P}$, et al. $\mathrm{p} 191$.

5 Maas PJ, van der Wal G, Haverkate I, et al. Euthanasia, physician-assisted suicide, and other medical practices involving the end of life in the Netherlands 1990-1995. N Engl J Med 1996:335: 1699-705.

6 Kuhse H, Singer P, Baume P, et al. p 192. 7 See reference 2: 4 .

8 Termination of Life on Request and Assisted Suicide (Review Procedures) Act 2001.

9 See reference 2: 264

10 See reference 2: 200

11 See reference 2: 249

12 A comparison of data from Australia and the Netherlands (see references 3 and 4 above) reveals the following: in Australia active voluntary euthanasia accounted for $1.7 \%$ of all deaths (an additional $0.1 \%$ involving assisted suicide) - in contrast with the Dutch position where active voluntary euthanasia was reported to be occurring at a rate of $1.8 \%$ of all deaths in 1990 ( $2.4 \%$ in 1995 ), but only $0.7 \%$ of all deaths in both years were attributed to killing without request. End-of-life decisions in medical practice in Flanders, Belgium:a nationwide survey. Lancet, $2000 ; 356: 1806-11$. This study found that active voluntary euthanasia occurred at a rate of $1.1 \%$ of all deaths (a further $0.1 \%$ accounting for assisted suicide) and killing without request occurred at a rate of $3.2 \%$ of all deaths, thus supporting the hypothesis that a policy of legal prohibition carries greater dangers for patients than does legalisation. For discussion see: $M$ Otlowski. The effectiveness of legal control of euthanasia: lessons from comparative law. Recht der Werkelijkheid [Journal of the Dutch/Flemish Association for Socio-Legal Studies] Special Issue, Elsevier, Regulating physician-negotiated death, 2002:133-55.

13 See reference 2: 262.

14 See reference 1: Kuhse $H$, Singer $P$, Baume $P$, etal.

15 See reference 2: 6.

16 See above, particularly reference 11 . 